\title{
Intracellular Lactate- and Pyruvate-Interconversion Rates Are Increased in Muscle Tissue of Non-Insulin-dependent Diabetic Individuals
}

\author{
A. Avogaro, ${ }^{\star}$ G. Toffolo, ${ }^{\ddagger}$ M. Miola, ${ }^{\star}$ A. Valerio, ${ }^{\star}$ A. Tiengo, ${ }^{\star}$ C. Cobelli, ${ }^{\ddagger}$ and S. Del Prato* \\ $*$ Cattedra di Malattie del Metabolismo, School of Medicine, and ${ }^{\ddagger}$ Department of Electronics and Informatics, University of Padova, \\ 35128 Padova, Italy
}

\begin{abstract}
The contribution of muscle tissues of non-insulin-dependent diabetes mellitus (NIDDM) patients to blood lactate appearance remains undefined. To gain insight on intracellular pyruvate/lactate metabolism, the postabsorptive forearm metabolism of glucose, lactate, FFA, and ketone bodies (KB) was assessed in seven obese non-insulin-dependent diabetic patients $\left(B M I=28.0 \pm 0.5 \mathrm{~kg} / \mathrm{m}^{2}\right)$ and seven control individuals $\left(\mathrm{BMI}=24.8 \pm 0.5 \mathrm{~kg} / \mathrm{m}^{2}\right)$ by using arteriovenous balance across forearm tissues along with continuous infusion of $\left[3-{ }^{13} \mathrm{C}_{1}\right]$-lactate and indirect calorimetry. Fasting plasma concentrations of glucose (10.0 \pm 0.3 vs. $4.7 \pm 0.2 \mathrm{mmol} / \mathrm{liter})$, insulin ( $68 \pm 5 \mathrm{vs} .43 \pm 6 \mathrm{pmol} / \mathrm{liter})$, FFA $(0.57 \pm 0.02$ vs. $0.51 \pm 0.02 \mathrm{mmol} / \mathrm{liter})$, and blood levels of lactate $(1.05 \pm 0.04$ vs. $0.60 \pm 0.06 \mathrm{mmol} / \mathrm{liter})$, and $\mathrm{KB}$ $(0.48 \pm 0.04$ vs. $0.29 \pm 0.02 \mathrm{mmol} /$ liter $)$ were higher in NIDDM patients $(P<0.01)$. Forearm glucose uptake was similar in the two groups $(10.3 \pm 1.4 \mathrm{vs} .9 .6 \pm 1.1 \mu \mathrm{mol} / \mathrm{min} / \mathrm{liter}$ of forearm tissue), while KB uptake was twice as much in NIDDM patients as compared to control subjects. Lactate balance was only slightly increased in NIDDM patients $(5.6 \pm 1.4 \mathrm{vs}$. $3.3 \pm 1.0 \mu \mathrm{mol} / \mathrm{min} / \mathrm{liter} ; P=\mathrm{NS}$ ). A two-compartment model of lactate and pyruvate kinetics in the forearm tissue was used to dissect out the rates of lactate to pyruvate and pyruvate to lactate interconversions. In spite of minor differences in the lactate balance, a fourfold increase in both lactate- $(44.8 \pm 9.0 \mathrm{vs}$. $12.6 \pm 4.6 \mu \mathrm{mol} / \mathrm{min} / \mathrm{liter})$ and pyruvate(50.4 \pm 9.8 vs. $16.0 \pm 5.0 \mu \mathrm{mol} / \mathrm{min} / \mathrm{liter})$ interconversion rates (both $P<0.01$ ) were found. Whole body lactate turnover, assessed by using the classic isotope dilution principle, was higher in NIDDM individuals (46 \pm 9 vs. $21 \pm 3 \mu \mathrm{mol} /$ $\mathrm{min} / \mathrm{kg} ; \boldsymbol{P}<\mathbf{0 . 0 1}$ ). Insights into the physiological meaning of this parameter were obtained by using a whole body noncompartmental model of lactate/pyruvate kinetics which provides a lower and upper bound for total lactate and pyruvate turnover $(\mathrm{NIDDM}=46 \pm 9-108 \pm 31$; controls $=$ $21 \pm 3-50 \pm 13 \mu \mathrm{mol} / \mathrm{min} / \mathrm{kg})$. In conclusion, in the postabsorptive state, despite a trivial lactate release by muscle, lactate- and pyruvate-interconversion rates are greatly enhanced
\end{abstract}

Address correspondence to Stefano Del Prato, M.D., Cattedra di Malattie del Metabolismo, Via Giustiniani, 2, 35128 Padova, Italy. Phone: 39-49-8212178; FAX: 39-49-8754179.

Received for publication 7 April 1994 and accepted in revised form 5 April 1996.

J. Clin. Invest.

(C) The American Society for Clinical Investigation, Inc.

0021-9738/96/07/108/08 \$2.00

Volume 98, Number 1, July 1996, 108-115 in NIDDM patients, possibly due to concomitant impairment in the oxidative pathway of glucose metabolism. This finding strongly suggest a major disturbance in intracellular lactate/pyruvate metabolism in NIDDM. (J. Clin. Invest. 1996. 98:108-115.) Key words: non-insulin-dependent diabetes mellitus - lactate metabolism - pyruvate metabolism • forearm metabolism $\bullet$ stable isotopes

\section{Introduction}

High fasting plasma glucose concentration in non-insulin-dependent diabetic (NIDDM) ${ }^{1}$ subjects is correlated with an increased rate of hepatic glucose production (1). The excess in hepatic glucose production has been attributed to accelerated gluconeogenesis (2), but the underlying mechanism(s) has not been identified. A direct substrate effect has been suggested (3). According to this hypothesis, plasma glucose taken up by peripheral tissues (muscles) mainly flows through anaerobic glycolysis (i.e., lactate release) since, in the postabsorptive conditions, glycogen synthesis is unlikely to occur and glucose oxidation is impaired (4). An increased activity of the Cori cycle (5) and of nonoxidative glycolysis (6) has been reported in obese NIDDM patients. Recent studies (3) have shown that rates of appearance in blood lactate and alanine were increased in NIDDM patients as compared to normal subjects, even though the tissues responsible for lactate release were not defined. In insulin-stimulated conditions blood lactate concentration is proportionally increased to the activation of whole body glucose disposal (7). Since under conditions of constant plasma glucose and insulin concentrations (glucose clamp) up to $80 \%$ of glucose uptake occurs in muscle (8), this tissue was believed to be a major contributor of lactate appearance in the circulation. Nevertheless, much controversy exists on the matter. Measurement of the arterio-venous balance across the forearm has led to both increased (3) or normal release (9) of lactate in NIDDM patients as compared to control individuals. Moreover, much criticism has been raised concerning interpretation of results obtained in studies using lactate tracers. Although arterio-venous balance of labeled lactate was used to assess the possible role of muscle as a lactate source for hepatic gluconeogenesis $(3,10)$, these data have been criticized on the ground that with the tracer method both lactate release and exchange of labeled lactate takes place with intracellular pyruvate. Therefore, the operativity of a lactate-pyruvate exchange would prevent equating uptake of the label with production and utilization of the compound (11-13), whereas lactate release is only reflected by the rate of conversion of pyruvate to lactate that exceeds that of lactate to pyruvate (14).

1. Abbreviations used in this paper: KB, ketone bodies; NIDDM, non-insulin-dependent diabetes mellitus. 
Therefore, the aim of the present study was to assess the interconversion rates of pyruvate to lactate and lactate to pyruvate in both NIDDM and control individuals to ascertain whether or not a disturbance in intracellular lactate metabolism is present in the muscle cells of hyperglycemic NIDDM patients. In addition, insights in the interpretation of rate of appearance of systemic lactate was sought by using a whole body model which takes into account interconversion rates.

\section{Methods}

\section{Subjects}

Seven obese NIDDM patients and seven normal control subjects (Table I) matched for age, gender, sex, and body mass index participated in the present study protocol. None of the NIDDM patients was treated with insulin. Four patients were taking sulfonylurea agents which were discontinued at least $4 \mathrm{~d}$ before each study. All subjects had normal renal and hepatic function and no symptoms or signs of vascular, heart, and pulmonary disease. No subject was taking medication known to adversely affect glucose metabolism. A weight-maintaining diet containing at least $200 \mathrm{~g}$ carbohydrate was consumed for at least $3 \mathrm{~d}$ before the study. The nature, purpose, and possible risks involved in the study were carefully explained to all subjects before obtaining their voluntary consent. The experimental protocol was approved by the local Ethical Committee.

\section{Protocol}

Subjects were admitted to the Unit of Metabolic Diseases of the University of Padova in the morning of the study, after a 10-12-h overnight fast. At 8:00 a.m., catheters were introduced percutaneously into the brachial artery and retrogradely into an ipsilateral deep forearm vein draining forearm muscle, respectively. The tip of the deep forearm vein catheter was advanced for a distance of 2 inches from the puncture site and could not be palpated in any of the subjects. Previous studies have documented that such catheter placement allows sampling of the muscle bed perfused by either the radial or ulnar artery (15). Catheter patency was maintained by a slow saline infusion. To exclude blood flow to the hand, a pediatric sphygmomanometric cuff was inflated about the wrist to $100 \mathrm{mmHg}$ above the systolic blood pressure for $3 \mathrm{~min}$ before and during each sampling interval. A third 18-gauge intravenous catheter was inserted into a contralateral superficial forearm vein for the initiation of a primed $(1.6 \mathrm{~g})$ continuous $(1.8 \mathrm{mg} / \mathrm{min})$ infusion of $\left[3-{ }^{13} \mathrm{C}_{1}\right]$-lactate that was kept constant for the ensuing $180 \mathrm{~min}$. After a $2 \mathrm{~h}$ isotope equilibration, blood samples were taken simultaneously from the brachial artery and the deep antecubital vein at 15 -min intervals over the last

Table I. Clinical and Basal Metabolic Characteristics of the Study Subjects

\begin{tabular}{lcc}
\hline & Controls & NIDDM \\
\hline Number & 7 & 7 \\
Sex $(\mathrm{M} / \mathrm{F})$ & $7 / 0$ & $7 / 0$ \\
Age $(\mathrm{yr})$ & $44 \pm 2$ & $50 \pm 3$ \\
Body mass index $\left(\mathrm{kg} / \mathrm{m}^{2}\right)$ & $24.8 \pm 0.5$ & $28.0 \pm 0.5^{*}$ \\
$\mathrm{HbA}_{1} \mathrm{c}(\%)$ & $4.6 \pm 0.1$ & $8.1 \pm 0.3^{\S}$ \\
Fasting plasma glucose $(\mathrm{mmol} / \mathrm{liter})$ & $4.7 \pm 0.2$ & $10.0 \pm 0.3^{\S}$ \\
Fasting plasma insulin $(\mathrm{pmol} /$ liter $)$ & $43 \pm 6$ & $68 \pm 5^{\S}$ \\
Fasting plasma free fatty acid (mmol/liter) & $0.51 \pm 0.02$ & $0.57 \pm 0.02^{*}$ \\
Fasting blood lactate $(\mathrm{mmol} /$ liter) & $0.60 \pm 0.06$ & $1.05 \pm 0.04^{\S}$ \\
Fasting blood ketone bodies ${ }^{\ddagger}(\mathrm{mmol} /$ liter $)$ & $0.29 \pm 0.02$ & $0.48 \pm 0.04^{\S}$
\end{tabular}

${ }^{*} P<0.05 ;{ }^{\ddagger}$ acetoacetate plus 3 -hydroxybutyrate; ${ }^{\S} P<0.01$ hour of the stable isotope infusion for determination of plasma hormone and substrate concentrations as well as ${ }^{13} \mathrm{C}$-lactate and ${ }^{13} \mathrm{C}$-pyruvate isotope ratios. Soon after blood collection, indocyanine green dye (Cardio-Green; Hyson, Westcott, and Dunning, Baltimore, MD) was infused through the arterial catheter at a rate of $2 \mathrm{ml} / \mathrm{min}$ while keeping the cuff inflated. After 4-5 min, a venous blood sample was taken to measure the plasma dye concentration. Forearm volume was measured by water displacement after completion of the study.

During the same time gas exchange measurements were performed using a metabolic measurement cart (Deltatrac; Datex, Milano, Italy) equipped by a canopy system. Oxygen content was continuously measured by electrochemical analysis, and carbon dioxide content was measured by an infrared analyzer. The amount of protein oxidized during the study was calculated from the urinary nitrogen excretion (16).

\section{Analytical procedures}

Plasma glucose was determined in duplicate on a Beckman glucose analyzer (Beckman Instruments, Fullerton, CA). Blood lactate, pyruvate, acetoacetate, 3-hydroxybutyrate concentrations were analyzed using a fluorometric methods (17). Plasma FFA concentrations were estimated using a microenzymatic method (18). Plasma concentrations of insulin and glucagon were assessed using standard radioimmunoassay as previously reported (19). Urinary nitrogen excretion was determined by the Kjeldahl method (20). Plasma flow was determined by green dye dilution and blood flow calculated based on the hematocrit (21).

${ }^{13} \mathrm{C}$-lactate and ${ }^{13} \mathrm{C}$-pyruvate were analyzed after deproteinization of blood sample as terbutyldimethylsilyl derivatives using gas chromatography mass spectrometry (22). The values of lactate and pyruvate ratios between labeled and unlabeled species, $R_{L}$ and $R_{P}$, in the sample were derived from the ion intensity ratios. For instance, for lactate, $\left[3-{ }^{13} \mathrm{C}_{1}\right]$ lactate and $\left[3-{ }^{12} \mathrm{C}_{1}\right]$ lactate are the labeled and unlabeled species with, respectively, ${ }^{13} \mathrm{C}$ - or ${ }^{12} \mathrm{C}$-isotope in position 3 . Species are thus defined with reference to specific atoms in specific positions, and, in deriving isotope ratios from the mass spectrum, we corrected analytically for interferences from the other atoms of the monitored ion.

The ratio $\mathrm{z}$ between tracer and tracee mass (or concentration) in the sample was evaluated from isotope ratio measurements (23). For instance, for lactate:

$z_{\mathrm{L}}(t)=\frac{l}{L}=\frac{R_{\mathrm{L}}(t)-R_{\mathrm{LN}}}{R_{\mathrm{I}}-R_{\mathrm{L}}(t)} \cdot \frac{1+R_{\mathrm{I}}}{1+R_{\mathrm{LN}}}$

where 1 is the tracer lactate concentration, that is the concentration in the sample of lactate from exogenous source; $\mathrm{L}$ is the tracee lactate concentration, that is the concentration of natural lactate from endogenous source; $R_{I}$ and $R_{L N}$ are the lactate isotope ratios in a sample of pure tracer and tracee, respectively.

\section{Calculations}

Regional model of lactate-pyruvate kinetics. The model of Fig. 1, originally proposed in the heart (22), has been used to describe lactatepyruvate kinetics in the forearm muscle tissue. The model assumes that lactate is produced and used in the tissue only through pyruvate, therefore the tracee lactate balance across the tissue equals the balance between interconversion fluxes of lactate into pyruvate, $\mathrm{F}_{\mathrm{PL}}$, and of pyruvate into lactate, $\mathrm{F}_{\mathrm{LP}}$

$\Phi\left(L_{\mathrm{A}}-L_{\mathrm{V}}\right)=F_{\mathrm{PL}}-F_{\mathrm{LP}}$

$\Phi$ is the tissue blood flow rate across the tissue, and $\mathrm{L}_{\mathrm{A}}$ and $\mathrm{L}_{\mathrm{V}}$ are the tracee lactate concentration at the arterial and venous side respectively.

By measuring tracer lactate balance across the tissue, $1_{\mathrm{A}}-1_{\mathrm{V}}$, and the tracer-to-tracee ratio of lactate and pyruvate, $\mathrm{z}_{\mathrm{L}}$ and $\mathrm{z}_{\mathrm{P}}$, the interconversion fluxes $\mathrm{F}_{\mathrm{PL}}$ and $\mathrm{F}_{\mathrm{LP}}$ can be calculated as 


\section{MUSCLE TISSUE}

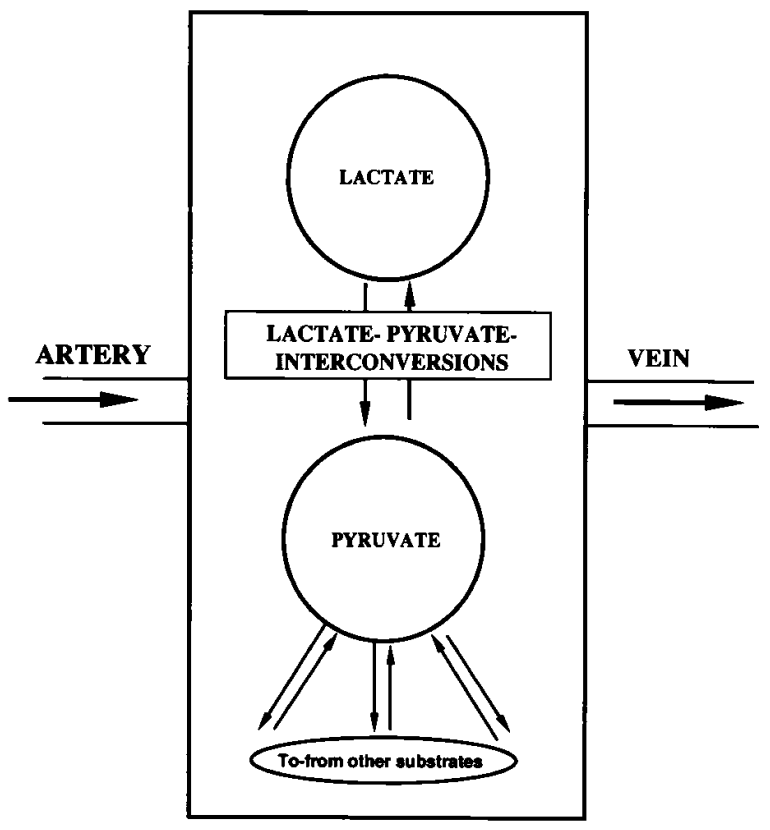

Figure 1. The compartmental model of lactate and pyruvate kinetics in the forearm tissue.

$F_{\mathrm{PL}}=\frac{\left[l_{\mathrm{A}}-l_{\mathrm{V}}\right]-z_{\mathrm{P}}\left[L_{\mathrm{A}}-L_{\mathrm{V}}\right]}{z_{\mathrm{L}}-z_{\mathrm{P}}} \Phi$

$F_{\mathrm{LP}}=F_{\mathrm{PL}}-\Phi\left[L_{\mathrm{A}}-L_{\mathrm{V}}\right]$

The derivation of Eqs. 2 and 3 is in Appendix.

In theory, the intracellular $\mathrm{z}_{\mathrm{L}}$ and $\mathrm{z}_{\mathrm{P}}$ should be used in equations (2) and (3). However, since the intracellular pool is nonaccessible, either the arterial or venous $\mathrm{z}_{\mathrm{L}}$ and $\mathrm{z}_{\mathrm{P}}$ are to be used. This implicitly assumes that they reflect intracellular ratio.

Whole-body model of lactate-pyruvate kinetics. The commonly used approach to quantify the turnover of lactate as seen from the accessible plasma pool defines the rate of appearance of lactate in plasma as

$R a=\frac{u}{z_{\mathrm{L}}}$

where $z_{\mathrm{L}}$ usually is the tracer to tracee ratio in the venous circulation.
However, lactate is de novo synthetized and irreversibly removed only after being interconverted into pyruvate, therefore the interpretation of $\mathrm{Ra}$ is difficult. To better understand the meaning of lactate Ra we adopted the general noncompartmental model of whole body lactate-pyruvate kinetics shown in Fig. 2. This model has previously been used to describe the kinetics of interconverting substrates, e.g., acetoacetate and 3-hydroxybutyrate (24). In this model, only the two accessible compartments are evidenced, representing plasma lactate and pyruvate, respectively. From plasma, lactate and pyruvate can each recirculate through indefinite number of compartments without being interconverted (fluxes $\mathrm{Rw}_{\mathrm{L}}, \mathrm{Rw}_{\mathrm{P}}$ ). Fluxes $\mathrm{R}_{\mathrm{LP}}$ and $\mathrm{R}_{\mathrm{PL}}$ between the two accessible pools represent lactate-pyruvate interconversions occurring either in accessible or in nonaccessible pools. Since pyruvate is de novo synthetized and irreversibly removed in the intracellular space where it also interconverts into lactate, both pyruvate, $\left(\mathrm{Ra}_{\mathrm{P}}\right)$, as well as lactate $\left(\mathrm{Ra}_{\mathrm{L}}\right)$ appear in plasma from de novo synthesis. Finally, both lactate and pyruvate are irreversibly removed from plasma $\left(\operatorname{Rd}_{\mathrm{L}}, \mathrm{Rd}_{\mathrm{P}}\right)$.

This general model allows us to gain insights into the meaning of lactate Ra: we show in the Appendix, that Ra (Eq. 4) equals $\mathrm{Ra}_{\mathrm{L}}$ plus the fraction of $\mathrm{Ra}_{\mathrm{P}}$ which appears in plasma as lactate after interconversion

$R a=R a_{\mathrm{L}}+R a_{\mathrm{P}} \frac{R_{\mathrm{LP}}}{R_{\mathrm{LP}}+R d_{\mathrm{P}}}$

From Eq. 5, one concludes that $\mathrm{Ra}$ overestimates $\mathrm{Ra}_{\mathrm{L}}$ and underestimates the total rate of appearance in plasma $\mathrm{Ra}_{\mathrm{L}}+\mathrm{Ra}_{\mathrm{P}}$ :

$R a_{\mathrm{L}} \leq R a \leq R a_{\mathrm{L}}+R a_{\mathrm{P}}$

Bounds of total lactate and pyruvate rate of appearance. If the pyruvate tracer to tracee ratio is also measured during the lactate tracer experiment, an upper bound for $\mathrm{Ra}_{\mathrm{L}}+\mathrm{Ra}_{\mathrm{P}}$ can be evaluated, since the ratio between the lactate tracer infusion rate $\mathrm{u}$ and pyruvate tracer to tracee ratio overestimates $\mathrm{Ra}_{\mathrm{L}}+\mathrm{Ra}_{\mathrm{P}}$ :

$\frac{u}{z_{\mathrm{P}}}=R a_{\mathrm{L}}+R a_{\mathrm{P}} \frac{R d_{\mathrm{L}}+R_{\mathrm{PL}}}{R_{\mathrm{PL}}} \geq R a_{\mathrm{L}}+R a_{\mathrm{P}}$

Summing up, from Eqs. 6 and 7 one concludes:

$\frac{u}{z_{\mathrm{L}}} \leq R a_{\mathrm{L}}+R a_{\mathrm{P}} \leq \frac{u}{z_{\mathrm{P}}}$

Therefore, upper and lower bounds for the total rate of appearance in plasma of lactate and pyruvate from de novo synthesis can be estimated from the lactate tracer experiment if tracer to tracee ratios of both lactate and pyruvate are measured. Notice that the quantification of all individual fluxes of the model in Fig. 2, in particular of the individual rates of appearance in plasma of lactate and pyruvate, re-

\section{RATES OF APPEARANCE}

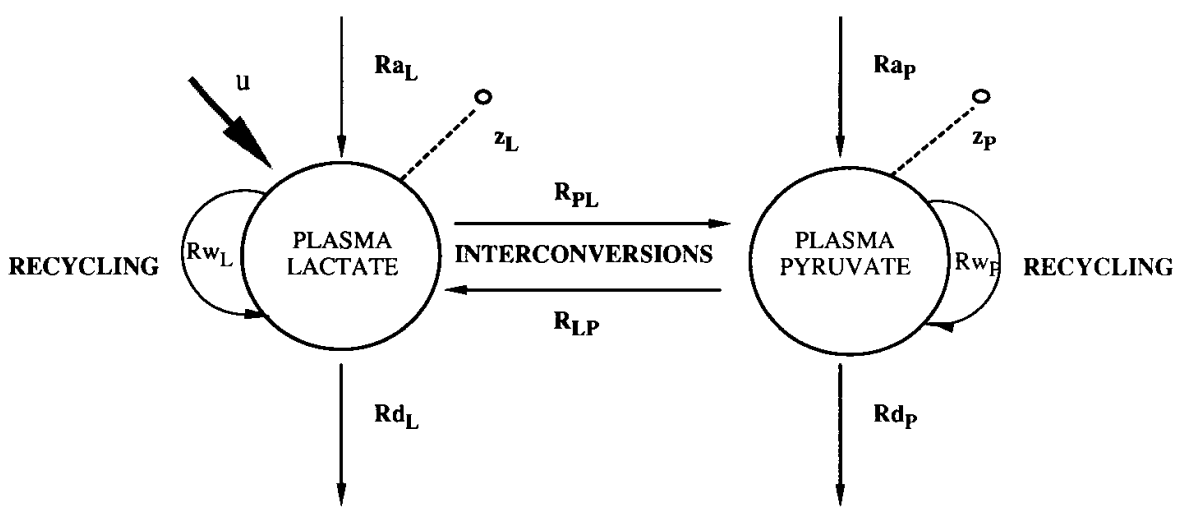

RATES OF DISAPPEARANCE
Figure 2. The whole-body noncompartmental model of lactate-pyruvate kinetics. 
Table II. Arterial and Vein Blood Lactate and Pyruvate Concentrations and Isotope Ratios $\left(z_{L}\right.$ and $\left.z_{P}\right)$ across the Forearm Tissues in Control Subjects and NIDDM Patients after an Overnight Fasting

\begin{tabular}{ccccc}
\hline & \multicolumn{2}{c}{ Controls } & \multicolumn{2}{c}{ NIDDM } \\
\cline { 2 - 3 } \cline { 5 - 5 } \cline { 4 - 5 } & Artery & Vein & Artery & Vein \\
\hline $\begin{array}{c}\text { Blood lactate } \\
\text { (mmol/liter) }\end{array}$ & $0.60 \pm 0.06$ & $0.72 \pm 0.07$ & $1.05 \pm 0.04^{\ddagger}$ & $1.21 \pm 0.19^{*}$ \\
$\begin{array}{c}\text { Blood pyruvate } \\
\quad(\mathrm{mmol} / \text { liter })\end{array}$ & $0.03 \pm 0.01$ & $0.03 \pm 0.01$ & $0.07 \pm 0.01^{*}$ & $0.06 \pm 0.01^{*}$ \\
$\mathrm{z}_{\mathrm{L}}$ & $0.021 \pm 0.003$ & $0.016 \pm 0.002$ & $0.021 \pm 0.004$ & $0.014 \pm 0.003$ \\
$\mathrm{z}_{\mathrm{P}}$ & $0.012 \pm 0.002$ & $0.009 \pm 0.002$ & $0.014 \pm 0.004$ & $0.008 \pm 0.003$
\end{tabular}

$* P<0.05 ;{ }^{*} P<0.01$.

quires a two tracer experiment, where labeled pyruvate is infused in addition to labeled lactate, and the four tracer to tracee ratios are measured in plasma.

Calculations. The balance across the forearm of glucose, lactate, FFA, and ketone bodies (KB) (acetoacetate plus 3-hydroxybutyrate) were calculated according to the Fick principle. Glucose and lipid oxidation were calculated from continuous calorimetric measurements (16). Data are given as mean $\pm \mathrm{SEM}$, and unpaired $t$ test was used to assess statistically significant differences.

\section{Results}

Glucose metabolism (Tables II and III). Both plasma glucose and plasma insulin were significantly higher in NIDDM patients as compared to control subjects. Forearm glucose uptake (Table III) was similar in the two groups (9.6 \pm 1.1 vs. $10.3 \pm 1.4$ $\mu \mathrm{mol} / \mathrm{min} /$ liter forearm tissue). By measurement of respiratory gas exchange a reduced rate of basal glucose oxidation $(5.5 \pm 0.5 \mu \mathrm{mol} / \mathrm{kg} / \mathrm{min})$ was found in NIDDM patients as compared to control individuals $(7.6 \pm 0.7 \mu \mathrm{mol} / \mathrm{kg} / \mathrm{min} ; P<0.05)$.

Regional lactate metabolism (Tables II and III and Fig. 3). Arterial and vein blood concentrations and isotope ratios of lactate and pyruvate across the forearm of the NIDDM patients and controls subjects are presented in Table II. After 10-12 h overnight fasting, blood lactate concentration was significantly higher in NIDDM $(1.05 \pm 0.04 \mathrm{mmol} / \mathrm{liter})$ than in control subjects $(0.60 \pm 0.06 \mathrm{mmol} /$ liter; $P<0.001)$. Forearm blood flow was similar in the two groups $(3.69 \pm 0.29$ and $3.51 \pm 0.32 \mathrm{ml} / \mathrm{min} / 100 \mathrm{ml})$. Lactate balance across the forearm

Table III. Glucose, Lactate, and Ketone Body Balances ( $\mu$ mol/ min/liter Muscle Tissue) across the Forearm Tissues in Control Subjects and NIDDM Patients after an Overnight Fasting

\begin{tabular}{lrc}
\hline & Controls & NIDDM \\
\hline Glucose balance & $10.3 \pm 1.4$ & $9.6 \pm 1.1$ \\
Lactate balance & $-3.3 \pm 1.0$ & $-5.6 \pm 1.4$ \\
Ketone body balance & $3.4 \pm 0.5$ & $7.8 \pm 1.4^{*}$ \\
FFA balance & $4.0 \pm 0.3$ & $4.5 \pm 0.2$
\end{tabular}

The positive sign indicates uptake while the negative one indicates release. ${ }^{*} P<0.05$.
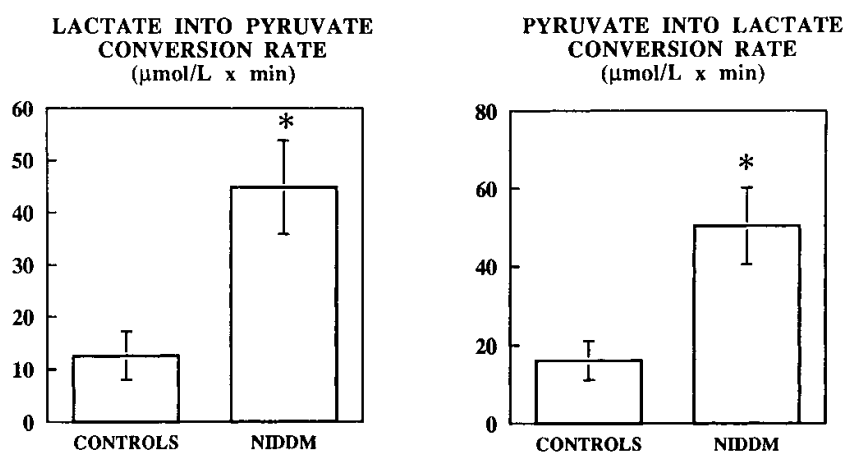

Figure 3. Interconversion rates of lactate to pyruvate (left) and pyruvate to lactate (right) occurring in the forearm muscle tissue of control individuals and NIDDM patients after a $10-12 \mathrm{~h}$ overnight fasting.

showed a slight release which was not significantly higher in NIDDM patients $(5.6 \pm 1.4$ vs. $3.3 \pm 1.0 \mu \mathrm{mol} / \mathrm{min} / \mathrm{liter}$ forearm tissue; $P=0.206)$. By using the two-compartment model shown in Fig. 1, the rates of interconversion from lactate into pyruvate $(44.8 \pm 9.0$ vs. $12.6 \pm 4.6 \mu \mathrm{mol} / \mathrm{min} / \mathrm{liter})$ and pyruvate into lactate $(50.4 \pm 9.8$ vs. $16.0 \pm 5.0 \mu \mathrm{mol} / \mathrm{min} /$ liter; both $P<$ 0.01 ) were three to fourfolds higher than in normal individuals (Fig. 3).

Systemic lactate metabolism. Systemic lactate appearance rate calculated according to the conventional equation for isotope dilution ( $\mathrm{Ra})$ was higher in NIDDM $(46 \pm 9 \mu \mathrm{mol} / \mathrm{min} / \mathrm{kg})$ than in control subjects $(21 \pm 3 \mu \mathrm{mol} / \mathrm{min} / \mathrm{kg} ; P<0.01)$. Upper and lower bounds for the total (lactate + pyruvate) rate of appearance, $\mathrm{Ra}_{\mathrm{L}}+\mathrm{Ra}_{\mathrm{P}}$, evaluated according to the new two accessible pool noncompartmental model of Fig. 2 were $21 \pm 3-$ $50 \pm 13 \mu \mathrm{mol} / \mathrm{min} / \mathrm{kg}$ and $46 \pm 9-108 \pm 31 \mu \mathrm{mol} / \mathrm{min} / \mathrm{kg}$ for control subjects and NIDDM patients, respectively.

Lipid and KB metabolism (Tables II and III). After 10-12 h overnight fast, plasma FFA concentration was higher in NIDDM patients $(0.57 \pm 0.02$ vs. $0.51 \pm 0.02 \mathrm{mmol} / \mathrm{liter} ; P<$ $0.05)$ than in controls with no difference in net FFA balance (Table III) between the two groups. This was associated with a significantly higher lipid oxidation $(3.5 \pm 0.2$ vs. $2.4 \pm 0.3 \mu \mathrm{mol} /$ $\mathrm{kg} / \mathrm{min} ; P<0.05$ ). Blood KB (acetoacetate +3 -hydroxybutyrate) concentration also was higher in NIDDM $(0.48 \pm 0.04$ vs. $0.29 \pm 0.02 \mathrm{mmol} /$ liter; $P<0.01)$ than in normal controls. Furthermore, an increased $\mathrm{KB}$ net uptake was apparent in NIDDM patients (Table III).

\section{Discussion}

Our results show that in obese NIDDM patients, after $10-12 \mathrm{~h}$ overnight fasting, postabsorptive glucose uptake is similar to that of normal individuals, probably as a consequence of the combined effects of hyperglycemia and hyperinsulinemia (6). Nevertheless, a peculiar disturbance of intracellular lactate/ pyruvate metabolism was apparent based upon the results obtained with the simultaneous determination of arterial and venous ${ }^{13} \mathrm{C}$-pyruvate and ${ }^{13} \mathrm{C}$-lactate tracer to tracee ratios. This approach indeed allowed us to interpret the data with a two-compartment model of lactate and pyruvate metabolism across the forearm muscle tissues (Fig. 1). This kinetic model has been previously applied for calculation of lactate metabolism and lactate-pyruvate interconversions in cardiac muscle of 
IDDM patients (22). In those patients we showed a higher lactate release by the heart of hyperglycemic IDDM subjects as a consequence of excessive flux rate of pyruvate into lactate not accompanied by concomitant reversal flux (22). On the contrary, in these NIDDM patients forearm lactate release was not significantly increased, due to a concomitant acceleration of the interconversion rate of lactate into pyruvate (Fig. 3).

For the calculation of the parameters related to lactate/ pyruvate kinetics, ideally, the intracellular tracer to tracee ratio should be used (25). Since the intracellular pool is nonaccessible, only the arterial or venous tracer to tracee ratio is available. The choice of the vein or the artery tracer to tracee ratio is based upon the assumption that one or the other more precisely reflects the intracellular ratio. We chose to use the venous tracer to tracee ratio because, as previously discussed by Saccà et al. (25), for substrates such as lactate and pyruvate, whose production and utilization proceed in the same domain, the arterial ratio would lead to overestimation of true tissue values. Venous tracer to tracee ratio is obviously lower than the arterial value, but, in principle, it can either under or overestimate the intracellular ratio. For sake of comparison, lactate/pyruvate kinetic parameters were calculated by using the arterial tracer to tracee ratio as well. In doing so, higher rates of lactate to pyruvate (NIDDM = 63.8 \pm 24.2 ; Controls = $11.7 \pm 5.1 \mu \mathrm{mol} / \mathrm{min} / \mathrm{liter}$ ) and pyruvate to lactate (NIDDM = $69.4 \pm 24.4$; Controls $=15.0 \pm 5.0 \mu \mathrm{mol} / \mathrm{min} /$ liter $)$ interconversion rates are obtained. Although different in absolute terms, the difference between NIDDM and control individuals is still present (68 and 73\% lower, respectively; both $P<0.05$ ), supporting a major disturbance in intracellular lactate metabolism in the muscle of NIDDM patients. Recent experimental findings on animal tissues $(26,27)$ have provided evidence that venous tracer to tracee ratio is higher than the intracellular one, with an even greater difference for the arterial tracer to tracee ratio. We derived hypothetical values for the intracellular tracer to tracee ratio by assuming a proportion between intracellular and venous tracer to tracee ratio similar to those found previously $(26,27)$. It is of interest that if these values are used in equations (1) and (2) the computed interconversion rates are still higher in NIDDM than in control individuals, and much closer to those obtained with venous $(\sim 5 \%$ less) than with arterial tracer to tracee ratio ( $\sim 20 \%$ higher $)$. Although these calculations remain merely speculative and artificial, they still support that the use of venous tracer to tracee ratio is likely to minimize the error due to nonaccessible intracellular lactate/pyruvate pool.

The reason for the high rate of lactate- and pyruvate-interconversion remains to be determined. The interconversion reaction occurs at the level of the pyruvate pool whose size is likely to be determined by the rate of glycolysis, rate of oxidation in the Krebs cycle, and equilibration between pyruvate and lactate and pyruvate and alanine through transamination processes. Among these mechanisms the rate of glycolysis and the pyruvate oxidation are likely to play a primary role, since alanine contribution to intracellularly produced pyruvate is $<10 \%$ (28).

Nonoxidative glycolysis is increased in NIDDM subjects (6). Furthermore, they are characterized by defective glucose oxidation $(4,6,29,30)$, an alteration inversely correlated with lipid oxidation (31). The high rate of lactate/pyruvate interconversion supports the hypothesis that lactate entry into the tricarboxylic acid cycle is impaired in hyperglycemic diabetic patients.
In this study, NIDDM patients, after a 10-12 h overnight fast, had higher plasma FFA concentration (Table II) and an increased rate of lipid oxidation. Furthermore KB concentration and uptake in the forearm of NIDDM patients was increased (Table II and III), suggesting that KB may contribute to increased intracellular acetyl-CoA/CoA ratio, another powerful inhibitor of the PDH activity (31).

It may be questioned whether or not an increased lactate/ pyruvate interconversion is a consequence of increased lactate concentration rather than a primary defect of muscle tissue of diabetic patients. However, the ratio between the interconversion rates and blood lactate concentration provides a much larger figure in NIDDM patients than in control individuals (48 vs. 16) suggesting an independent acceleration of the processes responsible for the shuttling between lactate and pyruvate.

The possibility that an increased rate of lactate/pyruvate interconversions is secondary to high intracellular concentration of lactate and pyruvate cannot be readily ruled out. Recent reports have documented that intracellular lactate concentration is increased in NIDDM patients (32). If that is the case, the increased interconversion rates of lactate and pyruvate might be seen as a sort of "futile cycle" possibly associated with a PDH defect, whereby energy is not dissipated or generated.

Finally, an effect of hyperglycemia may be considered. From this standpoint, it is of note that, in IDDM patients, net lactate balance and interconversion rates in the heart were normalized whenever measured after induction of euglycemia (21). Under condition of hyperglycemia and basal plasma insulin concentration the rise of intracellular levels of lactate can be accounted for by an increase in the glycolytic flux (33) without a simultaneous increase of the lactate flux exiting the cell. The latter may be the consequence of concomitant increase in $\mathrm{KB}$ utilization by the forearm tissues. Both organic acids may indeed compete for the same proton cotransport $(34,35)$. Finally, the increased availability of intracellular lactate will determine an enhanced lactate conversion into pyruvate, with the final result of a very high pyruvate/lactate interconversion rates.

Despite the disturbance in muscle lactate metabolism, no significant difference was detected in the lactate balance across the forearm muscle tissues of the NIDDM patients indicating that the contribution of local muscles to systemic blood lactate appearance is limited. Previous data on lactate forearm metabolism in both normal and NIDDM subjects are conflicting. Several reports have suggested that muscle forearm tissues is a significant contributor of lactate appearance in blood thus contributing to the release of gluconeogenetic precursors into the blood stream $(3,9,36,37)$. Such a possibility has been challenged by others (38-40) who proposed that not only muscle, splanchnic area, brain, erythrocytes, and skin produce lactate (4) but also adipose tissue represents a significant source of whole body lactate appearance (42-44). More recently, Jansson et al. (45) have documented that subcutaneous tissue is a major contributor of lactate release in the postabsorptive state and that this is further enhanced in obese subjects.

Systemic lactate appearance rate $(\mathrm{Ra})$, calculated by the conventional equation for isotopic dilution, was higher in the diabetic patients. This result agrees with previous reports (36, 37). However, the physiologic meaning of lactate $\mathrm{Ra}$ is not clear (11-14). Therefore, to gain a better understanding of the physiologic meaning of whole body Ra of lactate we described 
the system by mean of a whole body noncompartmental model which explicitly accounts for the lactate-pyruvate interconversions. The first result is that conventional lactate $\mathrm{Ra}$ actually represents the sum of two independent components: lactate appearance in plasma from de novo synthesis $\left(\mathrm{Ra}_{\mathrm{L}}\right)$ and a fraction of the pyruvate rate of appearance in plasma from de novo synthesis $\left(\mathrm{Ra}_{\mathrm{P}}\right)$ i.e., $R a_{\mathrm{P}} \cdot \frac{R_{\mathrm{LP}}}{R_{\mathrm{LP}}+R d_{\mathrm{P}}}$ (see Appendix), where $R_{\mathrm{LP}}$ is the interconversion flux of pyruvate into lactate, and $\mathrm{Rd}_{\mathrm{P}}$ is the pyruvate rate of disappearance. Thus, the physiologic interpretation of elevated values of $\mathrm{Ra}$ is difficult since it is a function of de novo synthesis of lactate, of the fraction of the pyruvate rate of appearance in plasma from de novo synthesis, of pyruvate to lactate interconversion rate, and rate of disappearance of pyruvate. A second result provided by the noncompartmental model consists in the definition of the upper and lower bounds for the total rate of appearance in plasma of lactate and pyruvate from de novo synthesis $R a_{\mathrm{L}}+$ $R a_{\mathrm{P}}$. Albeit lower and upper bounds for NIDDM patients are both higher than in control subjects, it is not possible to conclude that $R a_{\mathrm{L}}+R a_{\mathrm{P}}$ is indeed higher in the former. Additional experimental studies, i.e., with concomitant use of pyruvate tracer, are required to move from an interval to a point estimate and to solve the issue.

In conclusion, our results demonstrate that although muscle tissue of obese NIDDM patients with fasting hyperglycemia is unlikely to represent a major contributor to increased blood lactate appearance, in these individuals intracellular muscle lactate/pyruvate metabolism is deeply altered.

\section{Appendix}

Derivation of formulas for lactate-pyruvate kinetics at regional level. Denote by L, 1 the tracee (endogenous source) and tracer (exogenous source) concentration of lactate, which can be calculated from measurements of tracer to tracee ratio $z_{\mathrm{L}}=1 / L$ and total (tracer + tracee) concentration $\mathrm{c}_{\mathrm{L}}=1+\mathrm{L}$ as follows:

$L=c_{\mathrm{L}} \frac{1}{1+z_{\mathrm{L}}}$

$l=c_{\mathrm{L}} \frac{z_{\mathrm{L}}}{1+z_{\mathrm{L}}}$

Tracee lactate balance across the tissue equals the balance between interconversion fluxes (Fig. 1):

$\Phi\left(L_{\mathrm{A}}-L_{\mathrm{V}}\right)=F_{\mathrm{PL}}-F_{\mathrm{LP}}$

where $\Phi$ is the tissue blood flow rate across the tissue, subscripts $A$ and $\mathrm{V}$ denote arterial and venous side respectively, $\mathrm{F}_{\mathrm{LP}}$ is the tracee flux of pyruvate into lactate and $\mathrm{F}_{\mathrm{PL}}$ the flux of lactate into pyruvate.

Assuming a tracer steady state, a similar equation also holds for the tracer:

$\Phi\left(l_{\mathrm{A}}-l_{\mathrm{V}}\right)=f_{\mathrm{PL}}-f_{\mathrm{LP}}$

where $\mathrm{f}_{\mathrm{PL}}, \mathrm{f}_{\mathrm{LP}}$ are the tracer interconversion fluxes.

The tracer-tracee indistinguishability principle yields:

$\frac{f_{\mathrm{PL}}}{F_{\mathrm{PL}}}=z_{\mathrm{L}} ; \frac{f_{\mathrm{LP}}}{F_{\mathrm{LP}}}=z_{\mathrm{P}}$

which allows one to solve Eqs. $\mathrm{A} 3$ and $\mathrm{A} 4$ for $\mathrm{F}_{\mathrm{PL}}$ and $\mathrm{F}_{\mathrm{LP}}$ :
$F_{\mathrm{PL}}=\frac{\left[l_{\mathrm{A}}-l_{\mathrm{V}}\right]-z_{\mathrm{P}}\left[L_{\mathrm{A}}-L_{\mathrm{V}}\right]}{z_{\mathrm{L}}-z_{\mathrm{P}}} \Phi$

$F_{\mathrm{LP}}=F_{\mathrm{PL}}-\Phi\left[L_{\mathrm{A}}-L_{\mathrm{V}}\right]$

Derivation of formulas for lactate-pyruvate kinetics at whole body level. We refer here to the two accessible pool noncompartmental model of lactate pyruvate kinetics of Fig. 2. We start by writing the mass balance equations for the tracee and the tracer. For the tracee, the sum of the rates of appearance in plasma of lactate, $\mathrm{Ra}_{\mathrm{L}}$, and pyruvate, $R a_{P}$, equals the sum of the rates of disappearance, $\operatorname{Rd}_{L}$ and $\mathrm{Rd}_{\mathrm{p}}$ :

$R a_{\mathrm{L}}+R a_{\mathrm{P}}=R d_{\mathrm{L}}+R d_{\mathrm{P}}$

For the tracer, at steady state, the rate of infusion u equals the sum of the rates of disappearance of lactate, $\mathrm{rd}_{\mathrm{L}}$, and pyruvate, $\mathrm{rd}_{\mathrm{P}}$ :

$u=r d_{\mathrm{L}}+r d_{\mathrm{P}}$

The tracer-tracee indistinguishability principle yields:

$\frac{r d_{\mathrm{L}}}{R d_{\mathrm{L}}}=z_{\mathrm{L}} ; \frac{r d_{\mathrm{P}}}{R d_{\mathrm{P}}}=z_{\mathrm{P}}$

so that Eq. A9 can be written as:

$u=R d_{\mathrm{L}} z_{\mathrm{L}}+R d_{\mathrm{P}} z_{\mathrm{P}}$

We now derive an additional relationship between $\mathrm{z}_{\mathrm{L}}$ and $\mathrm{z}_{\mathrm{P}}$ by writing mass balance equations for the tracee and tracer in the pyruvate pool. For the tracee one has

$R a_{\mathrm{P}}+R_{\mathrm{PL}}=R d_{\mathrm{P}}+R_{\mathrm{LP}}$

where $\mathrm{R}_{\mathrm{PL}}, \mathrm{R}_{\mathrm{LP}}$ are tracee interconversion fluxes.

For the tracer, at steady state:

$r_{\mathrm{PL}}=r d_{\mathrm{P}}+r_{\mathrm{LP}}$

where $\mathrm{r}_{\mathrm{PL}}, \mathrm{r}_{\mathrm{LP}}$ are the tracer interconversion fluxes.

From the indistinguishability of tracer and tracee, $r_{\mathrm{PL}}=R_{\mathrm{PL}} z_{\mathrm{L}}$; $r d_{\mathrm{P}}=R d_{\mathrm{P}} z_{\mathrm{P}} ; r_{\mathrm{LP}}=R_{\mathrm{LP}} z_{\mathrm{P}}$, and Eq. $\mathrm{A} 13$ becomes:

$R_{\mathrm{PL}} z_{\mathrm{L}}=\left(R d_{\mathrm{P}}+R_{\mathrm{LP}}\right) z_{\mathrm{P}}$

and thus:

$z_{\mathrm{P}}=\frac{R_{\mathrm{PL}}}{R d_{\mathrm{P}}+R_{\mathrm{LP}}} z_{\mathrm{L}}$

By using Eq. A15, Eq. A11 becomes:

$u=\left(R d_{\mathrm{L}}+R d_{\mathrm{P}} \frac{R_{\mathrm{PL}}}{R d_{\mathrm{P}}+R_{\mathrm{LP}}}\right) z_{\mathrm{L}}$

Thus the commonly calculated lactate rate of appearance $\mathrm{Ra}=\mathrm{u} /$ $\mathrm{z}_{\mathrm{L}}$ can now be expressed in terms of lactate-pyruvate fluxes as:

$R a=\frac{u}{z_{\mathrm{L}}}=R d_{\mathrm{L}}+R d_{\mathrm{P}} \frac{R_{\mathrm{PL}}}{R d_{\mathrm{P}}+R_{\mathrm{LP}}}$

Finally, Ra can be related to the lactate and pyruvate rates of appearance $\mathrm{Ra}_{\mathrm{L}}$ and $\mathrm{Ra} \mathrm{a}_{\mathrm{P}}$ by using Eqs. $\mathrm{A} 8$ and $\mathrm{A} 12$ into Eq. $\mathrm{A} 17$ :

$$
\begin{aligned}
R a & =R a_{\mathrm{L}}+R a_{\mathrm{P}}-R d_{\mathrm{P}}+\frac{R d_{\mathrm{P}} R_{\mathrm{PL}}}{R d_{\mathrm{P}}+R_{\mathrm{LP}}} \\
& =R a_{\mathrm{L}}+R a_{\mathrm{P}}-\frac{R d_{\mathrm{P}}}{R d_{\mathrm{P}}+R_{\mathrm{LP}}}\left(R d_{\mathrm{P}}+R_{\mathrm{LP}}-R_{\mathrm{PL}}\right) \\
& =R a_{\mathrm{L}}+R a_{\mathrm{P}}-\frac{R d_{\mathrm{P}}}{R d_{\mathrm{P}}+R_{\mathrm{LP}}} R a_{\mathrm{P}} \\
& =R a_{\mathrm{L}}+R a_{\mathrm{P}} \frac{R_{\mathrm{LP}}}{R d_{\mathrm{P}}+R_{\mathrm{LP}}} .
\end{aligned}
$$


We have thus proved the equality between $\mathrm{Ra}$ and the sum of $\mathrm{Ra}_{\mathrm{L}}$ plus the fraction of $\mathrm{Ra}_{\mathrm{P}}$ which appears in plasma after interconversion, indicated as Eq. 5 in the section: Regional model of lactatepyruvate kinetics. In order to prove Eq. 6 of the same section, we derive from Eq. A14 the relationship between $\mathrm{z}_{\mathrm{L}}$ and $\mathrm{z}_{\mathrm{P}}$ :

$z_{\mathrm{L}}=\frac{R d_{\mathrm{P}}+R_{\mathrm{LP}}}{R_{\mathrm{PL}}} z_{\mathrm{P}}$

By using this relationships into Eq. A11:

$u=\left(R d_{\mathrm{L}} \frac{R d_{\mathrm{P}}+R_{\mathrm{LP}}}{R_{\mathrm{PL}}}+R d_{\mathrm{P}}\right) z_{\mathrm{P}}$

This equation allows one to express the ratio between the rate of lactate tracer infusion and pyruvate tracer to tracee ratio as a function of two accessible pool model fluxes:

$\frac{u}{z_{\mathrm{P}}}=R d_{\mathrm{L}} \frac{R d_{\mathrm{P}}+R_{\mathrm{LP}}}{R_{\mathrm{PL}}}+R d_{\mathrm{P}}$

By using Eq. A8 and A12 into Eq. A21, Eq. 6 is finally proved:

$$
\begin{aligned}
\frac{u}{z_{\mathrm{P}}} & =R d_{\mathrm{L}} \frac{R a_{\mathrm{P}}+R_{\mathrm{PL}}}{R_{\mathrm{PL}}}+R a_{\mathrm{L}}+R a_{\mathrm{P}}-R d_{\mathrm{L}} \\
& =R d_{\mathrm{L}} \frac{R a_{\mathrm{P}}}{R_{\mathrm{PL}}}+R a_{\mathrm{L}}+R a_{\mathrm{P}} \\
& =R a_{\mathrm{L}}+R a_{\mathrm{P}}\left(\frac{R d_{\mathrm{L}}}{R_{\mathrm{PL}}}+1\right) .
\end{aligned}
$$

\section{Acknowledgments}

We thank the nursing staff for their professional care of the subjects while on the research, and the technical staff for their superb contribution. We acknowledge Dr. Saula Vigili de Kreutzenberg for determining the green indocyanine dye.

This work was partially supported by Consiglio Nazionale delle Ricerche, Grants PF 4093.00457 and National Institutes of Health Grant RR-02176.

\section{References}

1. DeFronzo, R.A., E. Ferrannini, and D.C. Simonson. 1989. Fasting hyperglycemia in non-insulin dependent diabetes mellitus: contribution of excessive hepatic glucose production and impaired tissue glucose uptake. Metabolism. 38: 387-395.

2. Consoli, A., N. Nurjahan, F. Capani, and J. Gerich. 1989. Predominant role of gluconeogenesis in increased hepatic glucose production in NIDDM. Diabetes. 38:550-558.

3. Consoli, A., N. Nurjahan, J.J. Reilly, D.M. Bier, and J.E. Gerich. 1990. Mechanism of increased gluconeogenesis in non-insulin-dependent diabetes mellitus. Role of alterations in systemic, hepatic, and muscle lactate and alanine metabolism. J. Clin. Invest. 86:2038-2045.

4. Groop, L., R.C. Bonadonna, S. Del Prato, K. Ratheiser, K. Zick, E. Ferrannini, and R.A. DeFronzo. 1989. Glucose and free fatty acid metabolism in non-insulin-dependent diabetes mellitus: evidence for multiple sites of insulin resistance. J. Clin. Invest. 84:205-213.

5. Zawadzki, J.K., R.R. Wolfe, D.M. Mott, S. Lillioja, B. Howard, and C. Bogardus. 1988. Increased rate of Cori cycle in obese subjects with NIDDM and effect of weight reduction. Diabetes. 37:154-159.

6. Del Prato, S., R.C. Bonadonna, E. Bonora, G. Gulli, A. Solini, M. Shank, and R.A. DeFronzo. 1993. Characterization of cellular defects of insulin action in type 2 (non-insulin-dependent) diabetes mellitus. J. Clin. Invest. 91:484-494.

7. Trevisan, R., R. Nosadini, A. Avogaro, G. Lippe, E. Duner, P. Fioretto, R. Deana, P. Tessari, A. Tiengo, M. Velussi, A. Cernigoi, S. Del Prato, and G. Crepaldi. 1982. Type I diabetes is characterized by insulin resistance not only with regard to glucose, but also to lipid and aminoacid metabolism. J. Clin. Endocrinol. Metab. 62:1155-1162.

8. DeFronzo, R.A., E. Jacot, E. Jequier, E. Maeder, and P. Felber. 1981. The effect of insulin on the disposal of intravenous glucose: results from indirect calorimetry and hepatic and femoral vein catheterization. Diabetes. 30:1000-1007.
9. Capaldo, B., R. Napoli, P. Di Bonito, G. Albano, and L. Saccá. 1990. Glucose and gluconeogenic substrate exchange by the forearm skeletal muscle in hyperglycemic and insulin-treated type II diabetic patients. J. Clin. Endocrinol. Metab. 71:1220-1223.

10. Consoli, A., N. Nurjahan, J.J. Reilly, D.M. Bier, and J.E. Gerich. 1990. Contribution of liver and skeletal muscle to alanine and lactate metabolism in humans. Am. J. Physiol. 259:E677-E684.

11. Landau, B.R., and J. Wahren. 1992. The use of isotopes gone astray. Metabolism. 41:457-459.

12. Landau, B.R. 1993. Ketone and lactate metabolism: an exchange of conclusions. Metabolism. 42:260-262.

13. Sahlin, K. 1987. Lactate production cannot be measured with tracer techniques. Am. J. Physiol. 252:E439-E440.

14. Landau, B.R., and J. Wahren. 1993. Reply. Metabolism. 43:1500-1501.

15. Coles, D.R., K.E. Cooper, R.F. Mottram, and O.V. Occleshaw. 1958. The source of blood samples withdrawn from deep forearm vein via catheters passed upstream from median cubital vein. J. Physiol. (Lond.). 142:258-267.

16. Ferrannini, E. 1988. The theoretical basis of indirect calorimetry: a review. Metab. Clin. Exp. 37:287-301.

17. Lloyd, B., J. Burrin, P. Smythe, and K.G.M.M. Alberti. 1978. Enzymic fluorometric continuous flow assays for blood glucose, lactate, pyruvate, alanine, glycerol, and 3-hydroxybutyrate. Clin. Chem. 34:1727-1729.

18. Shimuzu, S., K. Inque, Y. Tani, and H. Yamada. 1979. Enzymatic microdetermination of serum free fatty acids. Anal. Biochem. 98:341-345.

19. Avogaro, A., A. Valerio, L. Gnudi, A. Maran, M. Zolli, E. Duner, A. Riccio, S. Del Prato, A. Tiengo, R. Nosadini. 1992. Ketone body metabolism in NIDDM. Effect of sulfonylurea treatment. Diabetes. 41:968-974.

20. Hawk, P.D. 1947. Kjeldahl method. In Practical Physiological Chemistry. Blakiston, Toronto. 814-822.

21. Dillion, R. 1965. Importance of hematocrit in interpretation of blood sugar. Diabetes. 14:672-678.

22. Avogaro, A., R. Nosadini, A. Doria, P. Fioretto, M. Velussi, C. Vigorito, L. Saccá, G. Toffolo, C. Cobelli, R. Trevisan, et al. 1990. Myocardial metabolism in insulin deficient diabetic humans without coronary artery disease. Am. J. Physiol. 258:E606-E618.

23. Cobelli, C., G. Toffolo, and D. Foster. 1992 Tracer-to-tracee ratio for analysis of stable isotope tracer data: link with radioactive kinetic formalism. Am. J. Physiol. 262:E968-E975.

24. Cobelli, C., R. Nosadini, G. Toffolo, A. McCulloch, A. Avogaro, and A. Tiengo. 1982. Model of the kinetics of ketone bodies in humans. Am. J. Physiol. 243:R7-R17.

25. Saccá, L., G. Toffolo, and C. Cobelli. 1992. V-A and A-V modes in whole body and regional kinetics: domain of validity from a physiological model. Am. J. Physiol. 263:E597-E606.

26. Chinkes, D.L., X.J. Zhang, J.A. Romijn, Y. Sakurai, and R.R. Wolfe. 1994. Measurements of pyruvate and lactate kinetics across the hindlimb and gut of anesthetized dogs. Am. J. Physiol. 257:E174-E182.

27. Large, V., M. Solovieiv, H. Brunengraber, and M. Beylot. 1995. Lactate and pyruvate isotopic enrichments in plasma and tissues of postabsorptive and starved rats. Am. J. Physiol. 268:E880-E888.

28. Newsholme, E.A., and A.R. Leech. 1983. Biochemistry for the Medical Science. John Wiley and Sons. New York.

29. Chen, Y.-I., A. Golay, A.L.M. Swislocki, and G.M. Reaven. 1987. Resistance to insulin suppression of plasma free fatty acid concentrations and insulin stimulation of glucose uptake in non-insulin dependent diabetes mellitus. $J$. Clin. Endocrinol. Metab. 64:17-21.

30. Lillioja, S., C. Bogardus, D.M. Mott, A.L. Kennedy, W.C. Knowler, and B.V. Howard. 1985. Relationship between insulin-mediated glucose disposal and lipid metabolism in man. J. Clin. Invest. 75:1106-1115.

31. Randle, J.P., P.B. Garland, and E.A. Newsholme. 1963. The glucose fatty acid cycle: its role in insulin sensitivity and the metabolic disturbances of diabetes mellitus. Lancet. i:785-789.

32. Vaag, A., P. Damsbo, O. Hoter-Nielsen, and H. Beck-Nielsen. 1992. Hyperglycemia compensates for the defects in insulin-mediated glucose metabolism and in the activation of glycogen synthase in the skeletal muscle of patients with type 2 (non-insulin-dependent) diabetes mellitus. Diabetologia. 35: 80-88.

33. Del Prato, S., A. Riccio, S. Vigili de Kreutzenberg, M. Dorella, A. Tiengo, and R.A. DeFronzo. 1995. Basal plasma insulin levels exerts a qualitative but not quantitative effect on glucose-mediated glucose uptake. Am. J. Physiol. 268:E1089-E1095.

34. Juel, C., A. Honig, and H. Pilegaard. 1991. Muscle lactate transport studied in sarcolemmal giant vescicles: dependence on fibre type and age. Acta Physiol. Scand. 143:361-365.

35. Beaudry, M., A. Duvallet, L. Thieulart, K. El Abida, and M. Rieu. 1991. Lactate transport in skeletal muscle cells: uptake in L6 myoblasts. Acta Physiol. Scand. 141:379-381.

36. Consoli, A., N. Nurjahan, J. Reilly, D.M. Bier, and J.E. Gerich. 1990. Contribution of liver and skeletal muscle to alanine and lactate metabolism in humans. Am. J. Physiol. 259:E677-E684.

37. Consoli, A., N. Nurjahan, J.E. Gerich, and L.J. Mandarino. 1992. Skeletal muscle is a major site of lactate uptake and release during hyperinsulinemia. 
Metabolism. 41:176-179.

38. Yki-Jarvinen, H., C. Bogardus, and J.E. Foley. 1990. Regulation of plasma lactate concentration in resting human subjects. Metabolism. 39:859-864.

39. Frayn, K.N., S.W. Coppack, and S.M. Humphreys. 1991. Glycerol and lactate uptake in human forearm. Metabolism. 40:1317-1319.

40. Jackson, R.A., R.D. Roshania, M.I. Hawa, B.M. Sim, and L. Di Silvio. 1986. Impact of glucose ingestion on hepatic and peripheral glucose metabolism in man: an analysis based on simultaneous use of the forearm and double isotope techniques. J. Clin. Endocrinol. Metab. 63:541-549.

41. Kreisberg, R.A. 1972. Glucose-lactate interrelations in man. N. Engl. J. Med. 287:132-137.
42. Frayn, K.N., S.W. Coppak, S.M. Humphreys, and P.L. Whyte. 1989. Metabolic characteristics of human adipose tissue in vivo. Clin. Sci. 76:509-516.

43. Jansson, P.-A., U. Smith, and P. Lönnroth. 1990. Evidence for lactate production by human adipose tissue in vivo. Diabetologia. 33:253-256.

44. Hagström, E., P. Arner, U. Ungerstedt, and J. Bolinder. 1990. Subcutaneous adipose tissue: a source of lactate production after glucose in humans. Am. J. Physiol. 258:E888-E893.

45. Jansson, P.-A., A. Larsson, U. Smith, and P. Lönnroth. 1994. Lactate release from the subcutaneous tissue in lean and obese men. J. Clin. Invest. 93 240-246. 